\title{
Assessing the hospital volume-outcome relationship in surgery: a scoping review
}

\author{
Mathieu Levaillant ${ }^{1,2^{*}}$, Romaric Marcilly ${ }^{1,3}$, Lucie Levaillant ${ }^{4}$, Philippe Michel ${ }^{5}$, Jean-François Hamel-Broza ${ }^{2}$, \\ Benoît Vallet ${ }^{1}$ and Antoine Lamer ${ }^{1}$
}

\begin{abstract}
Introduction: Many recent studies have investigated the hospital volume-outcome relationship in surgery. In some cases, the results have prompted the centralization of surgical activity. However, the methodologies and interpretations differ markedly from one study to another. The objective of the present scoping review was to describe the various features used to assess the volume-outcome relationship: the analyzed datasets, study population, outcome, covariates, confounders, volume modalities, and statistical methods.

Methods and analysis: The review was conducted according to a study protocol published in BMJ Open in 2020. Two authors (both of whom had helped to design the study protocol) screened publications independently according to the title, the abstract and then the full text. To ensure exhaustivity, all the papers included by each reviewer went through to the next step.

Interpretation: The 403 included studies covered 90 types of surgery, 61 types of outcome, and 72 covariates or potential confounders. 191 (47.5\%) studies focussed on oncological surgery and 37.8\% focussed visceral or digestive tract surgery. Overall, $86.6 \%$ of the studies found a statistically significant volume-outcome relationship, although the findings differed from one type of surgery to another. Furthermore, the types of outcome and the covariates were highly diverse. The majority of studies were performed in Western countries, and oncological and visceral surgical procedures were over-represented; this might limit the generalizability and comparability of the studies' results.
\end{abstract}

Keywords: Volume-outcome relationship, Hospital, Quality indicator, Surgery

\section{Introduction}

The hospital volume-outcome relationship in surgery has been extensively studied over the last decade. A significant relationship has been evidenced for various surgical procedures [1-4]; in all cases, a higher operating volume was associated with better patient outcomes. Given the consistency of this relationship from one setting to another, some researchers have recommended the creation of minimum volume thresholds in order to limit the number of centres with low levels of activity [5-7]. This

\footnotetext{
${ }^{*}$ Correspondence: mathieu.levaillant@gmail.com

${ }^{2}$ Methodologic and Biostatistics Department, CHU Angers, University

Angers, 4 rue Larrey, F-49000 Angers, cedex 9, France

Full list of author information is available at the end of the article
}

recommendation is also in line with the guidelines issued by the Expert Panel on Weight Loss Surgery [8]. These research findings prompted the French health authorities to consider the establishment of thresholds for oncological surgery in 2007 [9].

Even though the volume-outcome relationship appears to be relevant for a variety of surgical procedures and has prompted greater centralization [10], Morch et al's recent systematic review highlighted marked methodological differences between the studies in this field and suggested that further research should focus on the features used to assess the volume-outcome relationship [4]. These methodological disparities have been confirmed in a few publications; the significance of the volume-outcome relationship may depend on the way the outcome 
was explored, the covariates included in the model, the qualitative or quantitative categorization of the volume, and/or the type of statistical test applied [11, 12]. Hence, a study's methodology can have a direct impact on its conclusion $[13,14]$.

Most studies of the volume-outcome relationship have assessed mortality as the primary indicator. Although this is commonly assumed to be an essential outcome, mortality alone might not be sufficient for setting thresholds on surgical activity or for closing down low-volume centres - decisions that can have dramatic impacts on inequalities in health status and access to care [15]. In contrast, the potential lack of a significant relationship with volume does not mean that mortality is not of relevance for policy makers; it is acknowledged that this variable is positively associated with the length of hospital stay [16], recovery time [17], cost of the stay [18], related morbidity $[19,20]$ and (for cancer surgery) disease-free survival [21, 22]. Lastly, the identification of a positive volume-outcome relationship may not be enough to set thresholds. This doubt limits the reliability of this information as a basis for decision-making and the potential modification of organizational structures.

The above observations prompted us to consider that the volume-outcome relationship should be investigated more broadly. The objective of the present scoping review was to describe features that can be used to assess the volume-outcome relationship: the type of data analyzed, the study population, the study outcomes, the covariates and confounders considered, the hospital volume, and the interpretation of the results. Hence, this review of the volume-outcome relationship is intended to help researchers to choose outcomes and covariates of interest or even to identify new variables for investigation. Ultimately, this overview might help policy makers to understand the abundancy of the scientific literature and the breadth of this issue [23, 24].

\section{Method}

The present review's methodology (including the search and selection strategies and the analysis steps) is described elsewhere [25]. The review was conducted in six stages, as proposed by Arksey and O'Malley [26] and as subsequently modified by Levac et al. [27]. The present report complied with the Preferred Reporting Items for Systematic Reviews and Meta-Analyses (extension for scoping reviews) [28]. The main research question was as follows: how is the hospital volume-outcome relationship assessed in the field of surgery?

Suitable publications were identified according to the methodology developed by the Joanna Briggs Institute [29] (Table 1).
The PUBMED and Scopus databases were searched with the query shown in Table 2.

The publications were screened, selected and reviewed independently by two authors: a resident in public health (ML) and a medical informatics specialist (AL), both of whom had helped to draft the study protocol.

The literature was screened first by title and then by abstract, according to the inclusion and exclusion criteria (Table 3). Publications were included if they met all the inclusion criteria and none of the exclusion criteria. In each stage of the review, this method was tested on 10 publications. The two reviewers then met and checked that they agreed on the inclusion and exclusion decisions. All publications selected by either of the reviewers went through to the next step. The two reviewers' selections were not compared at the end of the title or abstract screening steps.

Lastly, the full text of selected publications were assessed for inclusion (Fig. 1). In the event of disagreement between the two reviewers, the final decision on inclusion was referred to a third reviewer (LL, who was also helped to design the study).

The reference lists of all selected publications were screened for additional studies meeting our inclusion and exclusion criteria.

The study data were extracted independently by the two reviewers, using a specific form (Supplementary Table 1). In the event of disagreement, the decision was referred to the third reviewer. After the data extraction form had been tested on the first 10 studies by both reviewers, it was validated as described in the study protocol [25]. No difficulties were encountered by either of the reviewers.

For each study, the following key data were extracted: first author, year of publication, country, study design, study objectives, the type of surgery, the database used to include patients, the inclusion and exclusion criteria, outcomes, confounders, statistical analyses, qualification of the volume variable, and conclusions. Using an inductive approach, the reviewers sorted the extracted data into the meta-categories listed in Table 4.

\section{Results}

\section{Description of the publications included}

We identified a total of 1010 publications in the Scopus database, and 1370 in the PUBMED database. After the removal of duplicates, 1621 publications remained. Next, 965 publications were excluded on the basis of the title, 81 were excluded on the basis of the abstract, and 172 were excluded on the basis of the full text (Fig. 1). No additional publications were included after screening the reference lists of those found in Scopus or PUBMED.

Four hundred three publications from 188 different journals were included in the review. The studies were 


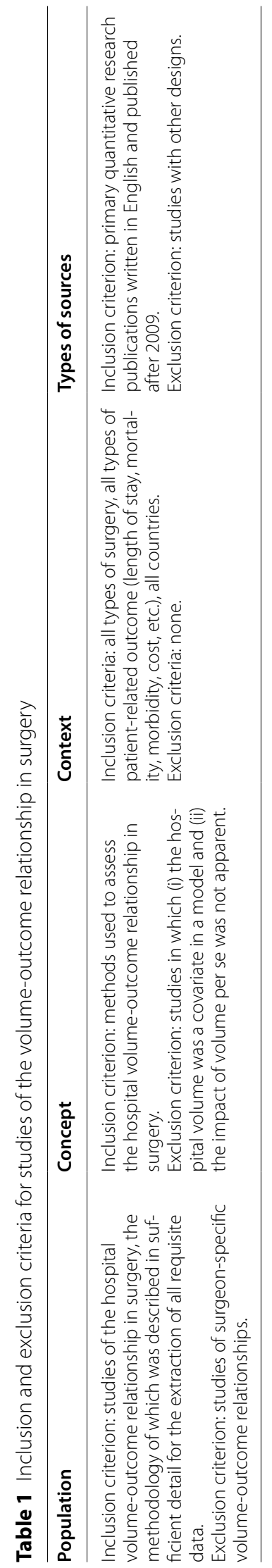


Table 2 Keywords and query used to search PUBMED and sCOPUS

\begin{tabular}{|c|c|}
\hline Database & Keywords and query \\
\hline PUBMED & $\begin{array}{l}\text { Keywords: Volume, outcome, hospital, surgery, } \\
\text { surgical, mortality, morbidity, cost } \\
\text { Query: ("Volume-outcome" OR "Volume-mortality" } \\
\text { OR ("hospital volume" AND ("outcome" OR "mortal- } \\
\text { ity" OR "morbidity" OR "cost"))) AND ("surgery" OR } \\
\text { "surgical") AND "hospital" NOT "surgeon"[TITLE] }\end{array}$ \\
\hline Scopus & $\begin{array}{l}\text { Keywords: Volume, outcome, hospital, surgery, } \\
\text { surgical, mortality, morbidity, cost } \\
\text { Query: TITLE-ABS-KEY ((“Volume-outcome" OR } \\
\text { "Volume-mortality" OR ("hospital volume" AND } \\
\text { ("outcome" OR" "mortality" OR "morbidity" OR } \\
\text { "cost"))) AND "surgery" AND"hospital") AND NOT } \\
\text { TITLE(“surgeon") AND (LIMIT-TO (PUBSTAGE, } \\
\text { "final")) AND (LIMIT-TO(DOCTYPE, "ar")) AND } \\
\text { (LIMIT-TO (LANGUAGE,"English")) }\end{array}$ \\
\hline
\end{tabular}

performed in 20 different countries, with more than half performed in the United States $(54.9 \% ; n=221$ ). The countries in which more than $3 \%$ of the studies were performed are represented in Fig. 2. Only 1 to $3 \%$ of the included studies were realised in Australia, Sweden, Finland, France, Korea, Spain, and this rate is lower than 1\% for Italy, Belgium, Norway, South Korea, Brazil, Italia and Switzerland.

There were very few multinational studies $(2.3 \% ; n=9)$.

The number of studies increased over time: a total of 24 papers were published during the period 2009-2011, whereas over 50 per year were published in 2018, 2019, and 2020.

\section{Data sources}

One hundred ninety-six different databases were used to study the volume-outcome relationship. More than half of these (54.75\%; $n=221$ ) were administrative databases, as defined by Levac et al. [31]. Nearly a third of them were patient or disease registers $(29.25 \% ; n=118)$, followed by claims databases $(11.00 \% ; n=44)$, health surveys $(2.25 \% ; n=9)$, and clinical trials data $(1.75 \% ; n=7)$.
Less than $1 \%$ of the included studies were based on data extracted from electronic health records.

\section{The surgical disciplines and procedures investigated}

Among the 403 studies reviewed, the most represented surgical discipline was visceral and digestive tract surgery $(37.75 \% ; n=152)$, followed by thoracic and cardiovascular surgery $(11.25 \% ; n=45)$, urology $(10.0 \% ; n=40)$, orthopaedic surgery $(9.50 \% ; n=38)$, vascular surgery $(8.0 \% ; n=32)$ and paediatric surgery $(5.5 \% ; n=22)$. Other specialties were explored in less than $5 \%$ of the studies.

Ninety distinct types of surgical activity were explored. Almost half of the studies concerned oncological indications $(191,47.5 \%)$. More than $5 \%$ of the publications studied pancreatic surgery $(5.24 \% ; n=21)$ followed by gastrectomy $(3.74 \% ; n=15)$, esophagectomy $(3.74 \%$; $n=15)$, aortic and mitral valve surgery $(3.74 \% ; n=15)$, rectal surgery $(3.74 \% ; n=15)$, hip surgery $(3.74 \%$; $n=15)$, lung surgery $(3.74 \% ; n=15)$, abdominal aortic aneurysms $(3.49 \% ; n=14)$, and cystectomy $(3.49 \%$; $n=14$ ). Other types of surgical activity were found in less than $3 \%$ of the studies.

In order to identify the patient populations undergoing surgery, $73.5 \%(n=296)$ of the publications used a version of the ICD. The majority of the publications that did not use the ICD $(65.1 \%)$ were based on patient or disease registers.

\section{Outcomes and hospital volume}

Hospital volume was expressed as a categorical variable only in $80.2 \%$ of the publications, as a continuous variable only in $4.3 \%$, and as a continuous variable and a categorical variable in $15.5 \%$.

Among the studies of volume as a categorical variable, nearly half (49.2\%) used quantiles. The other studies used literature definitions (19.8\%), statistically defined cut-offs $(5.8 \%)$, or other methods $(18.5 \%) .6 .6 \%$ of the studies assessed the volume as a categorical value in two or more ways.

Table 3 Inclusion and exclusion checklist

\begin{tabular}{lr}
\hline Criteria & Review Result \\
\hline Inclusion criteria: & $\square$ Yes $\boldsymbol{\square}$ No \\
Assessment of the hospital volume-outcome relationship in surgery & $\square$ Yes $\boldsymbol{\square}$ No \\
A precise description of the methodology (how the outcome was assessed, how the hospital volume was analyzed, and how the & $\square$ Yes $\boldsymbol{\square}$ No \\
statistical analysis was performed). & $\square$ Yes $\boldsymbol{\square}$ No \\
Exclusion criteria: & $\boldsymbol{\square}$ Yes $\boldsymbol{\square}$ No \\
Surgeon-specific volume-outcome relationship only & \\
Hospital volume used only as a covariate & \\
Publication as systematic review, qualitative study, editorial, letter to the editor, comment, narrative report, or any format other than & \\
primary quantitative research &
\end{tabular}




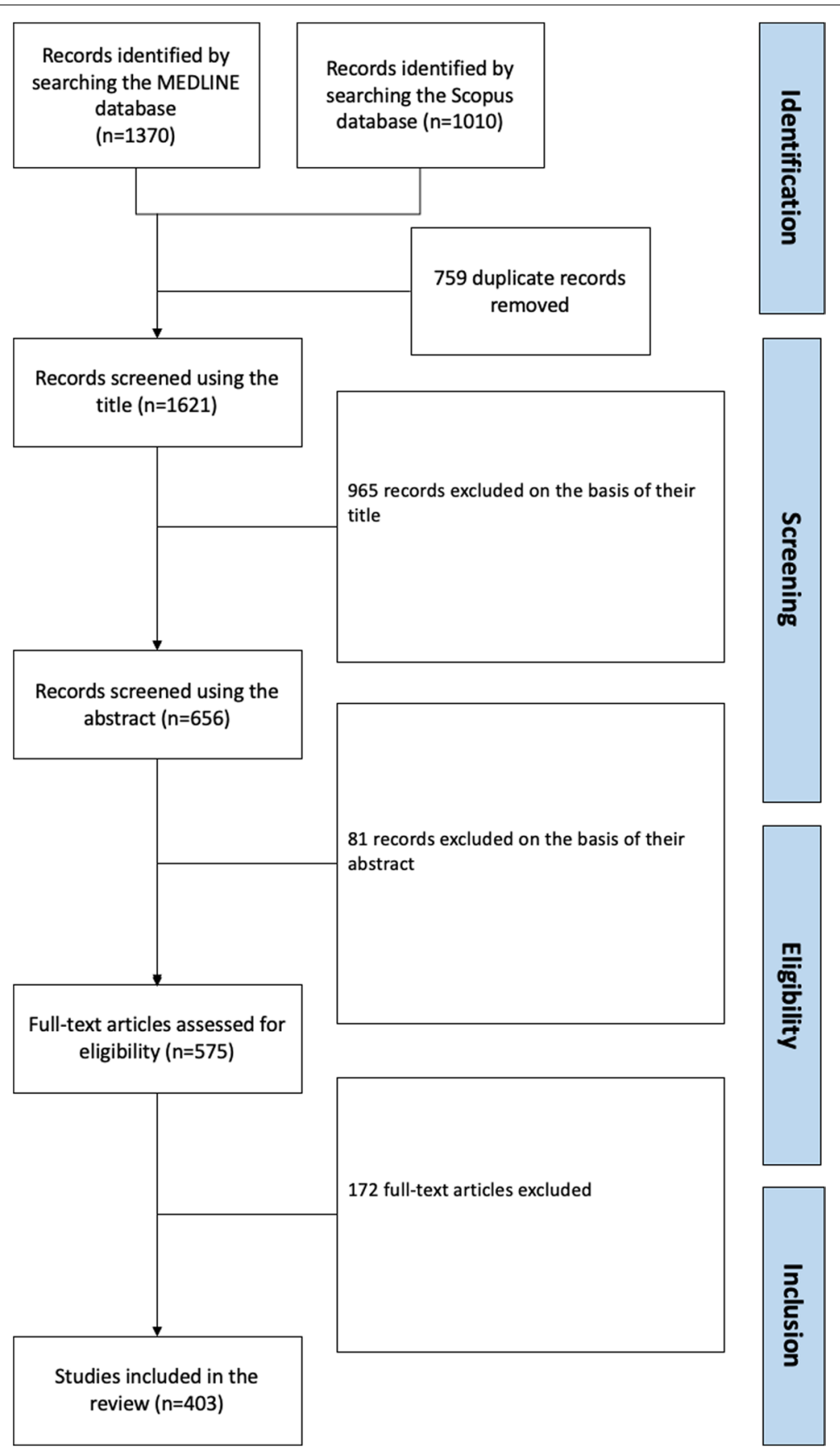

Fig. 1 Flow chart

Most of the included studies had more than one outcome. Mortality was the most frequently explored outcome $(79.9 \% ; n=321)$, followed by length of stay (32.0\%; $n=129)$, hospital readmission $(16.6 \% ; n=67)$, and cost $(16.1 \% ; n=65) .61 .0 \%(n=246)$ of the studies explored an outcome other than the four just mentioned. The most frequent of these were complications rates $(32.1 \% ; n=79)$, followed by failure-to-rescue (death after a major complication) $(7.0 \% ; n=17)$, specific oncological issues (5.5\%; $n=17)$, morbidity (3.5\%; $n=9)$, and discharge status $(2.7 \% ; n=7)$.

All 61 outcome variables are listed in Table 5. They were grouped into nine families: length of stay, mortality, readmission, oncological issues, cost, characteristics 


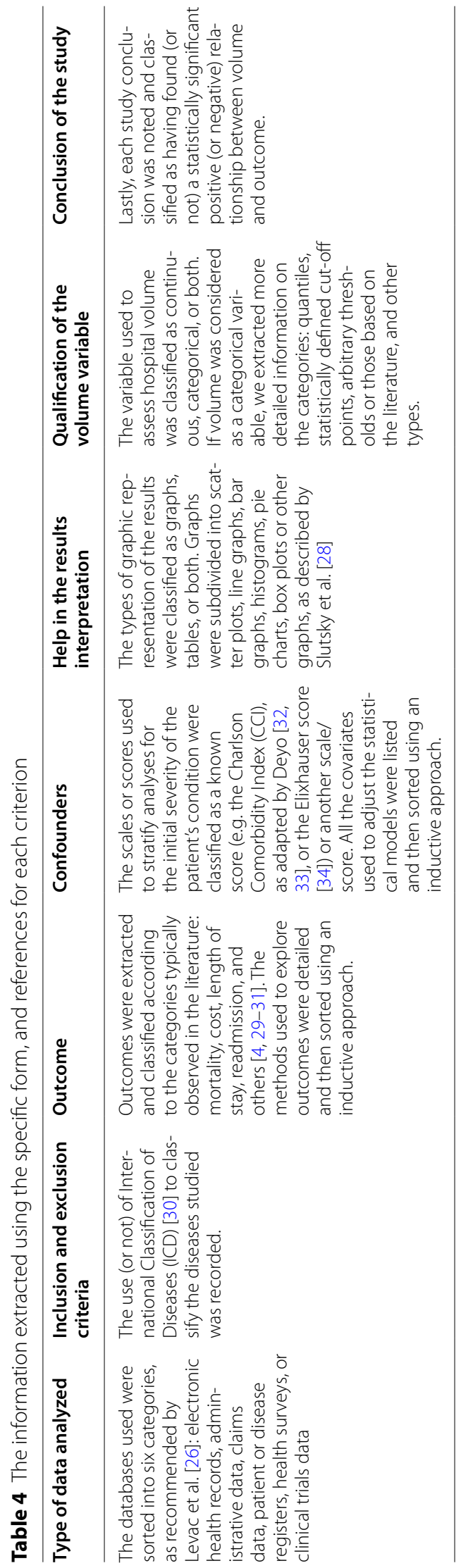




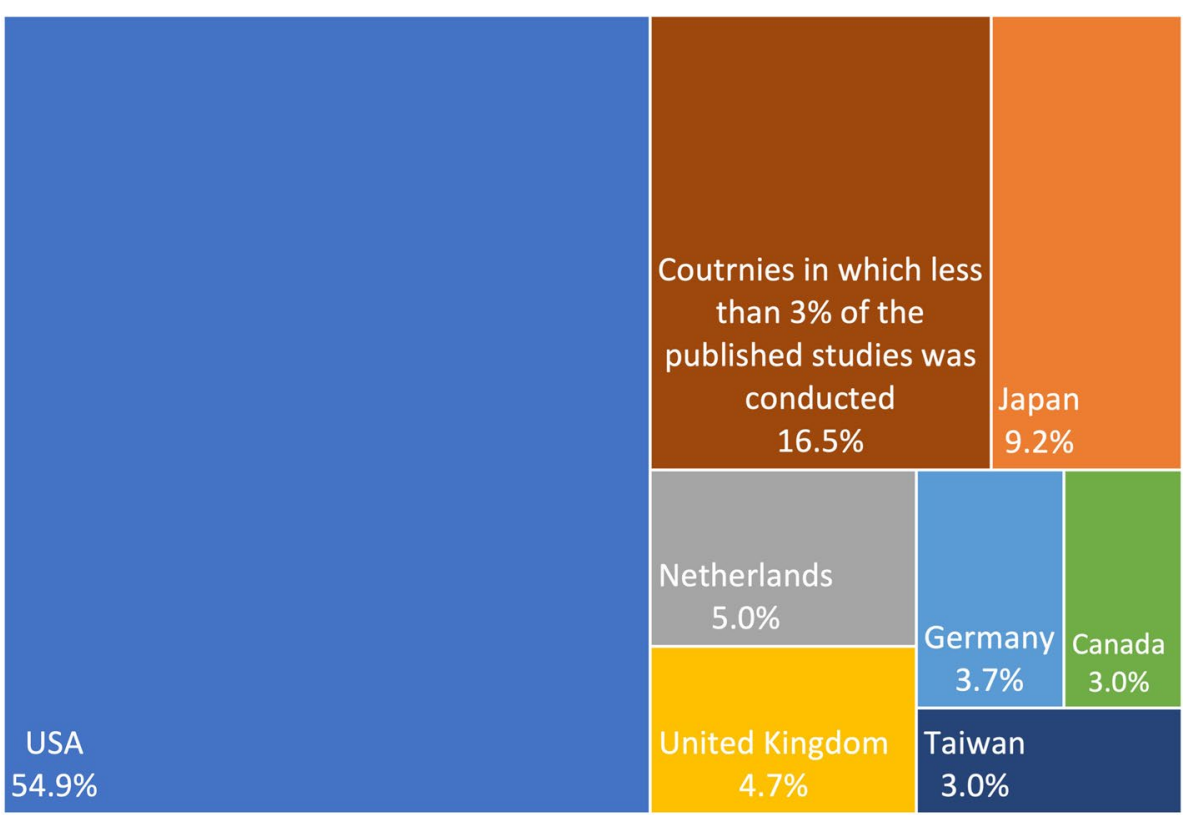

Fig. 2 Proportions of studies performed in each country

of the hospital stay, quality indicators, surgical complications, and medical complications.

We have not reported the proportions for each outcome because many studies used several of these (e.g. 30-day mortality and in-hospital mortality).

\section{Covariates included in the model, and assessment of the initial severity}

The 72 types of covariates used at least once for adjusting statistical models (listed in Table 6) were grouped into the following eight families, using an inductive approach: the patient's characteristics, the hospital's characteristics, clinical conditions, severity assessment, details of the disease, details of the surgery, details of the hospital stay, and post-operative events. Twenty five publications did not take into account any confounders when analyzing the hospital volume-outcome relationship. Five of the 25 studies (20\%) did not find a significant hospital volumeoutcome relationship. In contrast, only $12.6 \%$ of the studies that took account of potential confounders did not find this relationship.

\section{Statistically significant, positive volume-outcome relationships}

A statistically significant relationship between hospital volume and outcome was found in $86.6 \%(n=349)$ of the reviewed studies. Regardless of the volume modality, the type of outcome and the covariate(s) included in the model, $86.2 \%(n=347)$ of the studies that assessed mortality found a significant relationship. Depending on the way that the volume was assessed, either a greater hospital volume was significantly associated with a lower mortality rate or a group of hospitals with a higher volume had a lower mortality rate that a group with a lower volume. Furthermore, volume was significantly related the length of stay (in $89.1 \%$ of the studies; $n=359$ ), cost $(89.1 \% ; n=359)$, and hospital readmission $(79.1 \%$; $n=319$ ). A hospital volume-outcome relationship was also found in $87.3 \%(n=352)$ of the studies that explored at least one outcome other than those just listed.

This relationship was found only in 66.7\% $(n=269)$ of the studies performed in Korea, with values of $70.0 \%$ $(n=282)$ in Australia, 73.7\% $(n=295)$ in the Netherlands, and $75 \%(n=302)$ in Canada. For all other countries, the proportion of studies having found a statistically significant volume-outcome relationship was above $85 \%$.

The proportion of studies having found a statistically significant, positive volume-outcome relationship was similar for cancer indications (88\%) and other indications (85\%). The proportion was lower for paediatrics (68.2\%) and plastic surgery (75.0\%) but greater than $80 \%$ for other specialties (Fig. 3). A volume-outcome relationship was not evidenced for five types of surgery: benign prostate hyperplasia, cholangiocarcinoma, intra-arterial stroke treatment, intracranial aneurysms, and necrotizing enterocolitis. Four types of surgery (appendicectomy, colorectal resection, infantile hypertrophic pyloric stenosis or pancreas transplantation) featured a volumeoutcome relationship in less than $50 \%$ of the studies, and 6 types (liver transplantation, hysterectomy for cancer, 


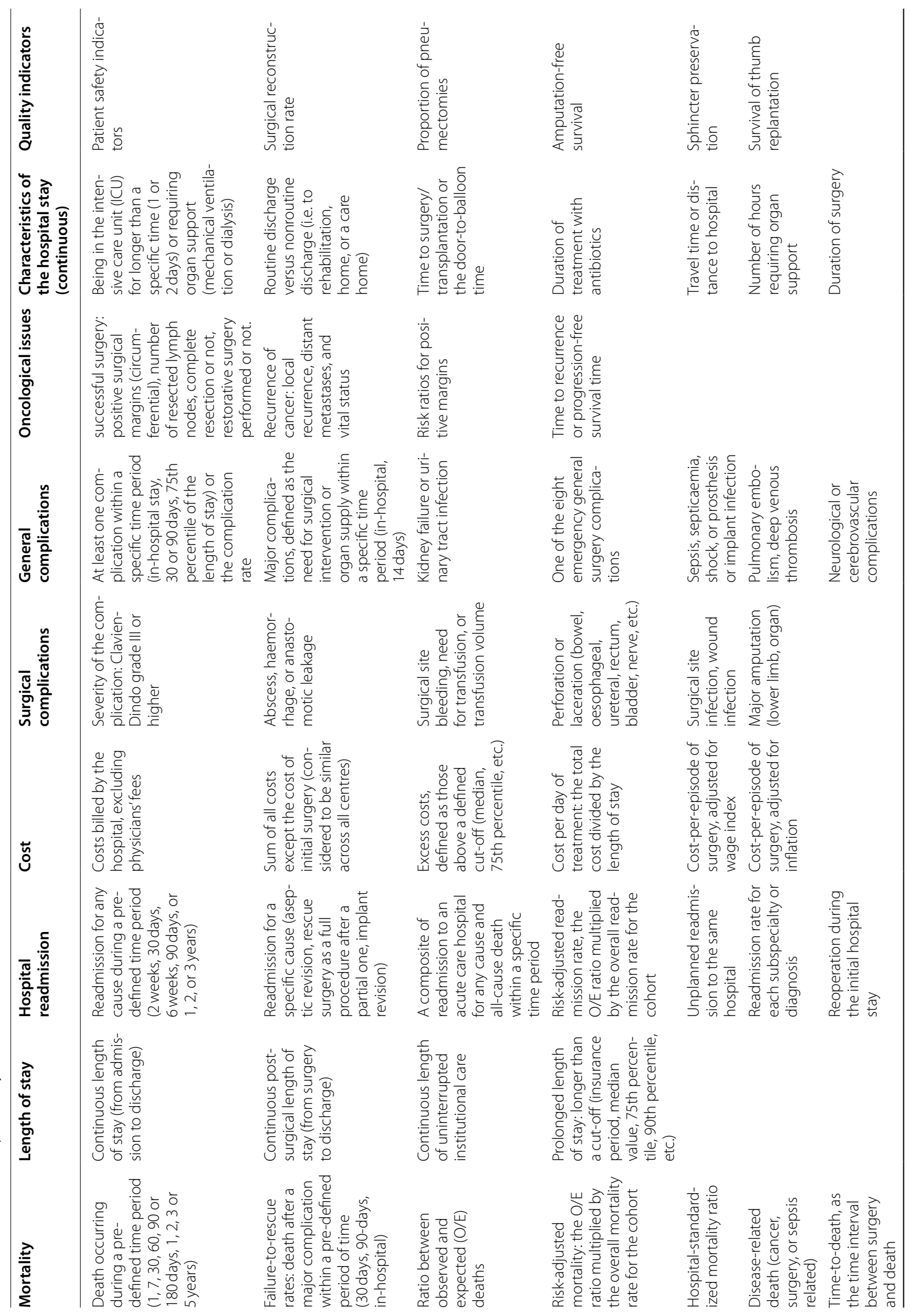




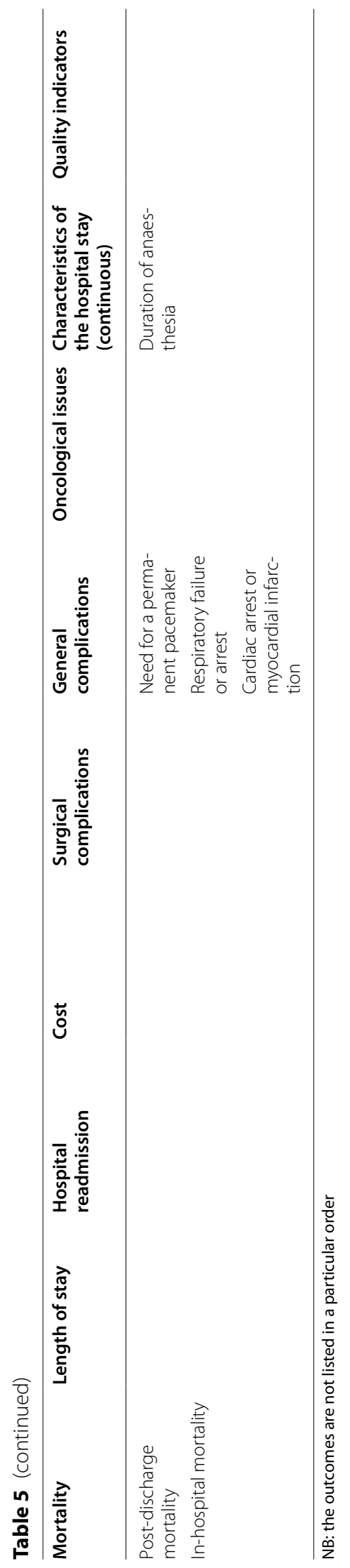




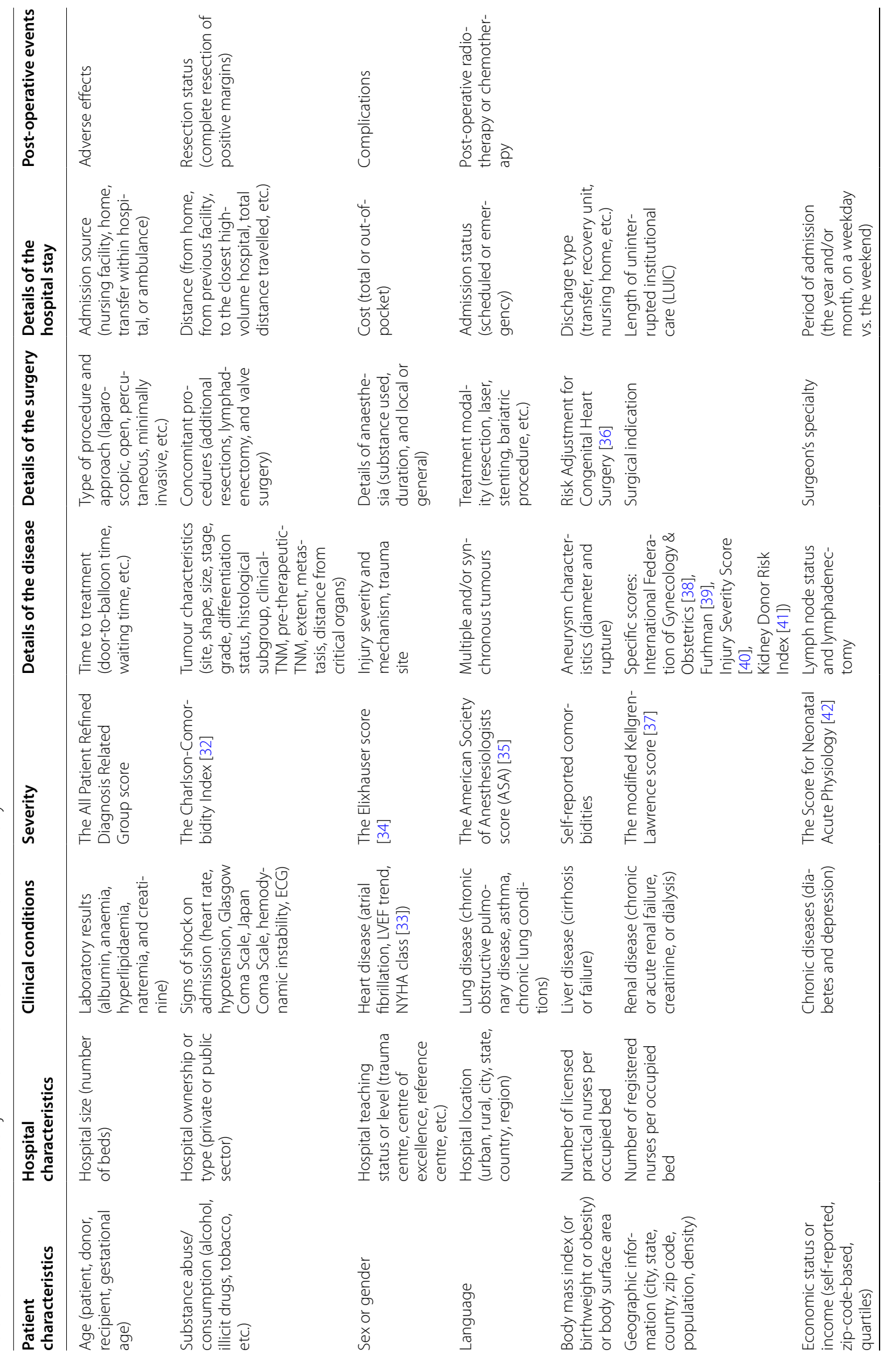




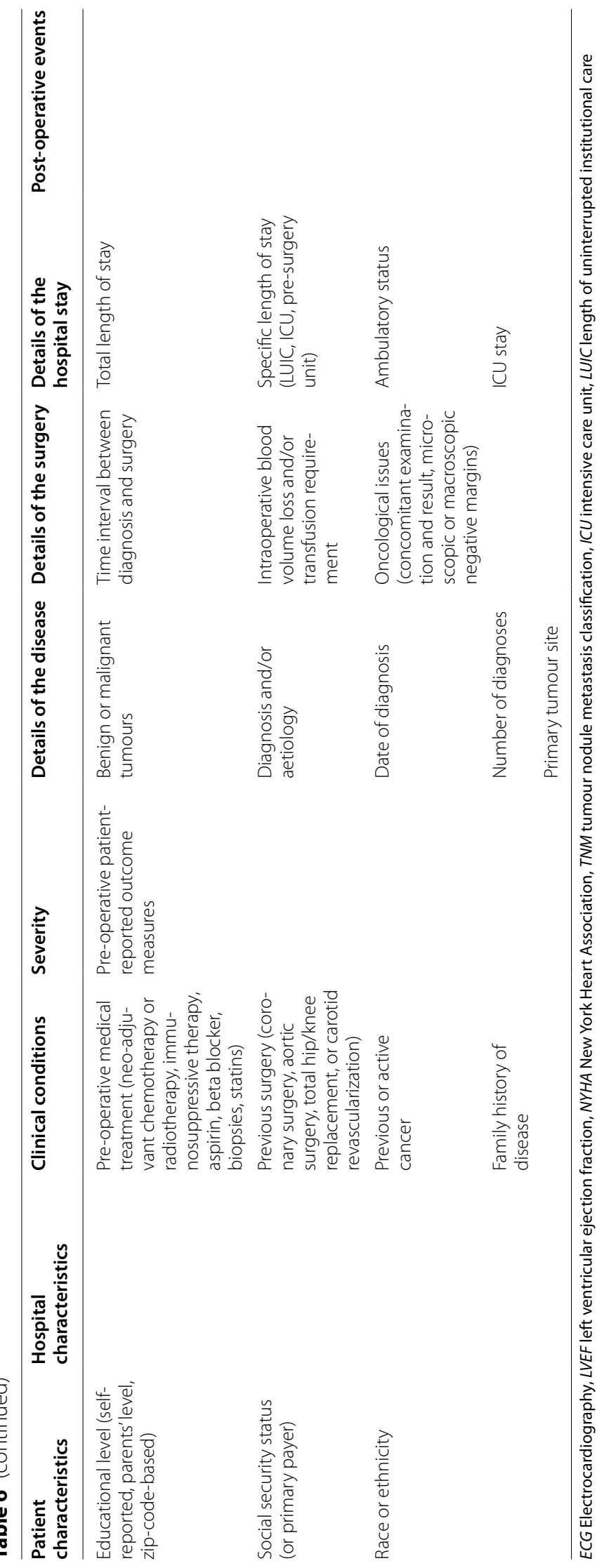




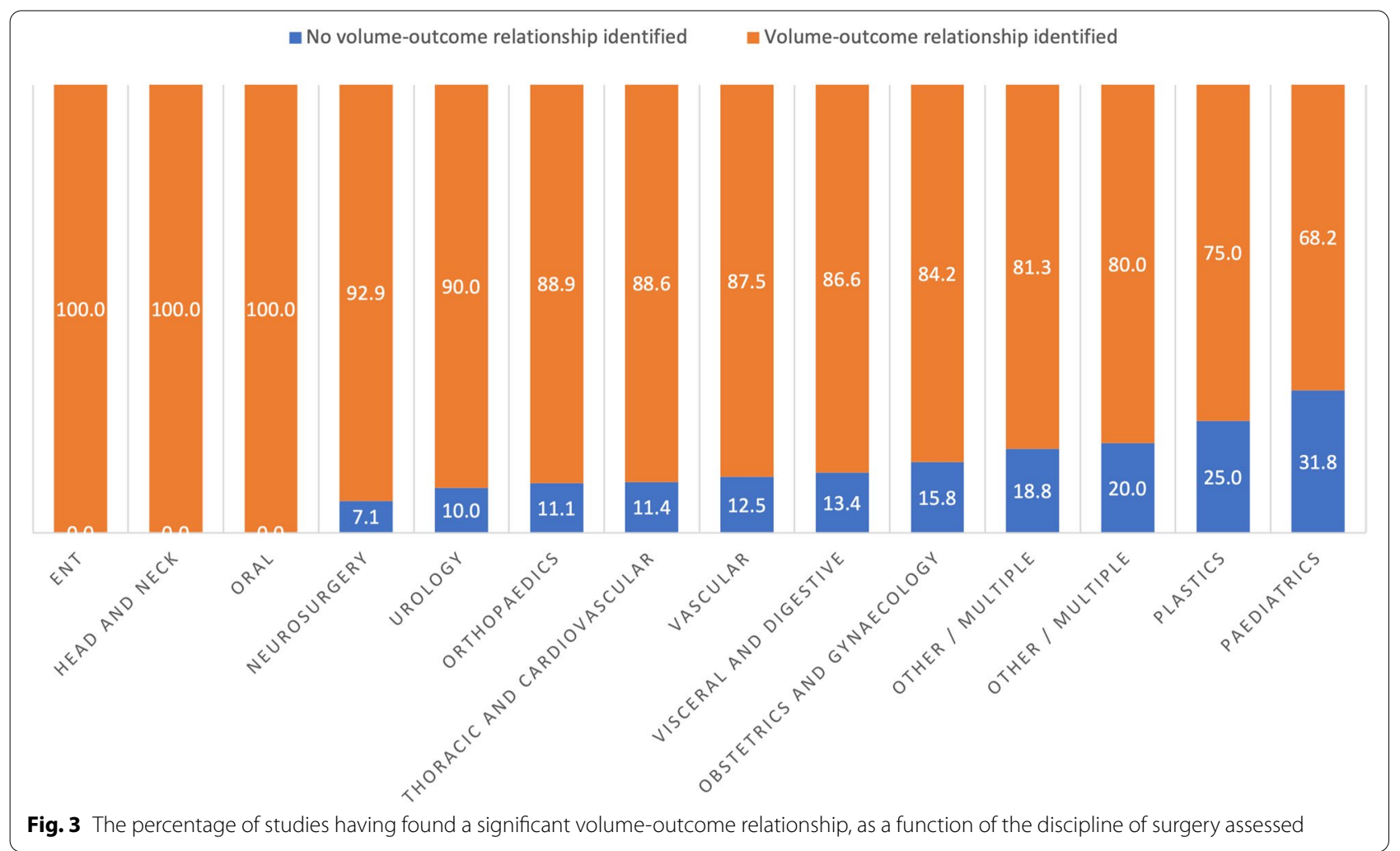

congenital diaphragmatic hernia, nephrectomy, total joint arthroplasty, and abdominal aortic aneurysm) featured a volume-outcome relationship in between 50 and $75 \%$ of the studies (Supplementary Figure 1).

\section{Discussion}

The objective of the present scoping review of the literature was to assess the ways in which the volume-outcome relationship was studied. Our main findings highlighted the diversity of the types of surgery, the types of outcome explored, and the method for exploring the volume-outcome relationship. The 403 studies included in the review variously assessed 90 types of surgery, 61 types of outcome, and 72 potential confounders.

Most of the studies (87.5\%) of the volume-outcome relationship had been performed in Western countries (as defined by Huntington [43]). More than half of all the included studies were based on administrative databases (54.8\%), even though the latter do not always describe all the patients treated in a given centre. In fact, some administrative databases only describe patients with social security coverage or other types of health insurance, and some (particularly in the USA) even describe only patients covered by a particular private healthcare provider. A proportion of the patient population specifically concerned by the volume-outcome issue might therefore have been excluded from these studies. In countries with low success rates, it would be interesting to look at why quality varied. The high proportion of Western countries may limit the degree to which the studies' data can be extrapolated.

Nearly $50 \%$ of the studies assessed cancer surgery (47.5\%), and a third assessed visceral or digestive tract surgery (37.8\%). This distribution might not reflect actual levels of activity. By way of an example, only $8.1 \%$ of hospital stays for surgery in France in 2019 were for an oncological indication (vs. $47.5 \%$ in the present review). The corresponding values are $27.2 \%$ for orthopaedic surgery (9\% in the review), $17.9 \%$ for ophthalmology (less than 5\% here), $13.1 \%$ for digestive tract or visceral study $(37.8 \%$ here), $9.1 \%$ for urology (10\% here), and 5.5\% for cardiovascular surgery ( $11.3 \%$ here); hence, the proportions found here do not match the activity data [44, 45]. Even when comparing our review's results with the activity in the US reported by Stanford HealthCare in the United States in 2009 , only $12.7 \%$ of operations concerned the digestive tract $(37.8 \%$ here), with $2.3 \%$ for the urinary tract (10\% here) and $15.2 \%$ for the cardiovascular system (11.3\% here) [46]. Studies that found a positive volumeoutcome relationship for rare, complex, specific types of surgery must be interpreted with caution, since they may not reflect surgical activity in general. 
Although $86.6 \%$ of the reviewed studies found a statistically significant volume-outcome relationship, the results differed from one type of surgery to another. For example, a significant relationship was found in $70 \%$ of the studies of paediatric surgery and not at all for five specific types of surgery (benign prostate hyperplasia, cholangiocarcinoma, intra-arterial stroke treatment, intracranial aneurysms, and necrotizing enterocolitis).

Our review highlighted a high degree of diversity among the outcomes measured and the covariates included in statistical analyses. Even though almost $80 \%$ of the studies investigated mortality as one of their outcomes, the way it was assessed modified the end results. For example, some studies looked at 5-year mortality among a population of elderly patients in which life expectancy can be a major source of bias, whereas other looked at 1-day mortality. Cost (explored in $16.1 \%$ of the studies) always has a particular context and depends on the country in which it is studied. Indeed, the share of a given cost paid by the patient may differ markedly in the USA vs. France. Moreover, patient outcomes may be interlinked because nursing facilities in some countries (but not in others) have incentives to hospitalize residents [47].

This heterogeneity can be viewed as both a strength and a limitation, and a few studies have shown the results depend on the variable or analytical method used [13, 48]. In 2015, Yu et al. showed that categorization of volume as either a continuous variable, in quartiles or as $\mathrm{k}$-means yielded different relationships with the outcome [14]. In 2018, Bernard et al. reported that four different regression models gave significantly different results for the same datasets [49].

Covariates also have a major impact on the assessment of the volume-outcome relationship. A recent study of cholangiocarcinoma resection showed that the relationship was no longer significant after adjustment for the distance travelled [50].

Volume may not be the only issue to be considered. For example, Mukhtar et al. compare high-activity years with low/medium-activity years in a San Francisco hospital year over a 15 -year period; neither the complication rate nor the mortality rate depended on the surgical volume [51]. These results are suggestive of a learning curve effect. Indeed, centres that increased their volume yearon-year sometimes had better outcomes than centres with absolute volumes that were higher but decreased year-on-year [52, 53].

The study populations in high-volume centres and low-volume centres are probably not the same, and thus should be taken into account in the analytical model. Indeed, Liu et al's 2017 study of cancer surgery showed that patient attendance at low-volume centres was associated with a shorter travelling distance, residence in a rural area, and the absence of neoadjuvant therapy but not with the severity of their disease [54]. In 2017, Gani et al. showed that ethnic minorities, elderly patients, and patients with many comorbidities may have more difficulty accessing high-volume centres, which increases inequalities in access to care [15].

Even though the great majority of studies (in almost all surgical fields and all countries) found a volumeoutcome relationship, those that explored centralization showed that having only high-volume centre had adverse effects and might not improve patient outcomes. Stitzenberg et al. reported that a marked increase in travelling distance observed after the centralization of pancreatic surgery posed a significant obstacle to accessing quality care [55] and increased inequalities in care access for specific populations - mainly in rural states [56]. Dimick et al. even suggested that given the size of the USA and the numbers of some types of surgery, nationwide local access to a high-volume facility is impossible [57].

The great variety of outcomes and covariates used to assess the hospital volume-outcome relationship, the high predominance of studies in Western countries, and the over-representation of oncological, visceral and digestive tract surgery may limit the generalizability of the studies' results. Given the many different ways in which this relationship has been explored, policymakers should be very careful when using the conclusions of specific studies to modifying healthcare facility maps.

This review suffered from several limitations. Firstly, the study's design as a scoping review prevented us from evaluating the methodological quality of each study included. Secondly, our predefined categories may not have been precise enough to analyse each type of study. Indeed, the database categories, the types of surgery and the statistical methods could have been more precise. However, with a view to overcoming this limitation, the extraction grid was first tested on 10 studies. Thirdly, our literature search was limited to two electronic databases (PUBMED and Scopus) and the search terms selected may not be exhaustive. Hence, other relevant publications in other databases, or presenting none of the included keywords would have been missed [58]. Fourthly, our review was limited to the scientific literature and thus did not cover the pricing data used by policy makers to take decisions about healthcare facility mapping. Lastly, we reviewed the hospital volume-outcome relationship for surgery in general. Hence, our results may be relevant from the hospital perspective rather than that of individual surgeons.

The present review is the first to provide an exhaustive overview of how volume-outcome relationship has been explored and how relevant criteria can be selected as a 
function of a study's objective. Results showed that even if most of the study showed a significant volume-outcome relationship, every feature of the analysis provide a different information. In consequence, before using such results to adapt a health facility mapping, policy-makers should perform a specific study on the surgery and territory of interest. In order to help them with such analysis, this review tries to provide a set of tools for investigating the volume-outcome relationship that can be adapted depending on the desired goal.

\section{Supplementary Information}

The online version contains supplementary material available at https://doi. org/10.1186/s12874-021-01396-6.

Additional file 1 : Supplementary Figure 1. The percentage of studies in which a significant volume-outcome relationship, as a function of the type of surgery assessed.

Additional file 2: Supplementary Table 1. Extraction data form.

Additional file 3. Reference list of the studies included in the review.

\section{Acknowledgements}

Not applicable.

\section{Authors' contributions}

ML led the design and conceptualization of this work and developed the search strategy with AL. ML and AL screened the publications, read all of the included publications and filled out the extraction grid. ML and AL wrote the manuscript. LL was the third reviewer when consensus on the inclusion of a publication was required. RM and JFHB served as experts for application of the protocol's methodology. RM, LL, JFHB, PM and BV provided critical comment on the manuscript and revised the final version. All authors approved the final manuscript.

\section{Funding}

This research did not receive any specific funding from agencies or organizations in the public, commercial, or not-for-profit sectors.

\section{Availability of data and materials}

The datasets used and/or analysed during the current study available from the corresponding author on reasonable request.

\section{Declarations}

\section{Ethics approval and consent to participate}

Literature reviews are considered to be secondary analyses and do not require ethical approval. The present findings will be disseminated through several channels, such as conferences and peer-reviewed journals. This research did not require patient consent as no patient were concerned.

\section{Consent for publication}

Not applicable.

\section{Competing interests}

The authors declare no conflicts of interest.

\section{Author details}

1 Univ. Lille, CHU Lille, ULR 2694 - METRICS: Évaluation des technologies de santé et des pratiques médicales, F-59000 Lille, France. ${ }^{2}$ Methodologic and Biostatistics Department, CHU Angers, University Angers, 4 rue Larrey, F-49000 Angers, cedex 9, France. ${ }^{3}$ Inserm, CIC-IT 1403, F-59000 Lille, France. ${ }^{4}$ Department of Paediatric Endocrinology and Diabetology, Angers University Hospital, Angers, France. ${ }^{5}$ Hospices Civils de Lyon ; Université Claude Bernard
Lyon 1, Research on Healthcare Performance (RESHAPE), INSERM U1290, Lyon, France.

Received: 18 April 2021 Accepted: 9 September 2021

Published online: 09 October 2021

\section{References}

1. El Amrani M, Clement G, Lenne X, Rogosnitzky M, Theis D, Pruvot F-R, et al. The impact of hospital volume and Charlson score on postoperative mortality of proctectomy for rectal cancer: a nationwide study of 45,569 patients. Ann Surg. 2018;268(5):854-60.

2. El Amrani M, Clement G, Lenne X, Farges O, Delpero J-R, Theis D, et al. Failure-to-rescue in patients undergoing pancreatectomy: is hospital volume a standard for quality improvement programs? Nationwide analysis of 12,333 patients. Ann Surg. 2018;268(5):799-807.

3. Pasquer A, Renaud F, Hec F, Gandon A, Vanderbeken M, Drubay V, et al. Is centralization needed for esophageal and gastric cancer patients with low operative risk?: a nationwide study. Ann Surg. 2016;264(5):823-30.

4. Morche J, Mathes T, Pieper D. Relationship between surgeon volume and outcomes: a systematic review of systematic reviews. Syst Rev. 2016;5(1):204

5. Dixon M, Mahar A, Paszat L, McLeod R, Law C, Swallow C, et al. What provider volumes and characteristics are appropriate for gastric cancer resection? Results of an international RAND/UCLA expert panel. Surgery. 2013;154(5):1100-9.

6. Choi H, Yang S-Y, Cho H-S, Kim W, Park E-C, Han K-T. Mortality differences by surgical volume among patients with stomach cancer: a threshold for a favorable volume-outcome relationship. World J Surg Onc. 2017:15(1):134.

7. Gutacker N, Bloor K, Cookson R, Gale CP, Maynard A, Pagano D, et al. Hospital surgical volumes and mortality after coronary artery bypass grafting: using international comparisons to determine a safe threshold. Health Serv Res. 2017;52(2):863-78.

8. Lim LRB, Blackburn GL, Jones DB. Benchmarking best practices in weight loss surgery. Curr Probl Surg. 2010;47(2):79-174.

9. Décret n²007-388 du 21 mars 2007 relatif aux conditions d'implantation applicables à l'activité de soins de traitement du cancer et modifiant le code de la santé publique (dispositions réglementaires) - Légifrance. [cited 2020 Dec 29]. Available from: https://www.legifrance.gouv.fr/loda/ id/JORFTEXTO00000275848/2020-12-29/

10. Vonlanthen R, Lodge P, Barkun JS, Farges O, Rogiers X, Soreide K, et al. Toward a consensus on centralization in surgery. Ann Surg. 2018;268(5):712-24

11. Christian CK, Gustafson ML, Betensky RA, Daley J, Zinner MJ. The volumeoutcome relationship: don't believe everything you see. World J Surg. 2005:29(10):1241-4

12. Halm EA, Lee C, Chassin MR. Is volume related to outcome in health care? A systematic review and methodologic critique of the literature. Ann Intern Med. 2002;137(6):511.

13. Kim W, Wolff S, Ho V. Measuring the volume-outcome relation for complex hospital surgery. Appl Health Econ Health Policy. 2016;14(4):453-64.

14. YU T-H, Tung Y-C, Chung K-P. Does categorization method matter in exploring volume-outcome relation? A multiple categorization methods comparison in coronary artery bypass graft surgery surgical site infection. Surg Infect. 2015;16(4):466-72.

15. Gani F, Azoulay D, PawlikTM. Evaluating trends in the volume-outcomes relationship following liver surgery: does regionalization benefit all patients the same? J Gastrointest Surg. 2017;21(3):463-71.

16. Modrall J, Minter R, Minhajuddin A, Eslava-Schmalbach J, Joshi G, Patel S, et al. The surgeon volume-outcome relationship: not yet ready for policy. Ann Surg. 2018;267(5):863-7.

17. Balentine CJ, Naik AD, Robinson CN, Petersen NJ, Chen GJ, Berger $\mathrm{DH}$, et al. Association of high-volume hospitals with greater likelihood of discharge to home following colorectal surgery. JAMA Surg. 2014;149(3):244-51

18. Sutton JM, Hoehn RS, Ertel AE, Wilson GC, Hanseman DJ, Wima K, et al. Cost-effectiveness in hepatic lobectomy: the effect of case volume on mortality, readmission, and cost of care. J Gastrointest Surg. 2016;20(2):253-61. 
19. Gourin CG, Stewart CM, Frick KD, Fakhry C, Pitman KT, Eisele DW, et al. Association of hospital volume with laryngectomy outcomes in patients with larynx cancer. JAMA Otolaryngol Head Neck Surg. 2019;145(1):62-70.

20. Odagiri H, Yasunaga H, Matsui H, Matsui S, Fushimi K, Kaise M. Hospital volume and adverse events following esophageal endoscopic submucosal dissection in Japan. Endoscopy. 2017;49(4):321-6.

21. Borowski DW, Bradburn DM, Mills SJ, Bharathan B, Wilson RG, Ratcliffe AA, et al. Volume-outcome analysis of colorectal cancer-related outcomes. $\mathrm{Br}$ J Surg. 2010;97(9):1416-30.

22. van der Werf LR, Cords C, Arntz I, Belt EJT, Cherepanin IM, Coene P-PLO, et al. Population-based study on risk factors for tumor-positive resection margins in patients with gastric cancer. Ann Surg Oncol. 2019;26(7):2222-33.

23. Grant MJ, Booth A. A typology of reviews: an analysis of 14 review types and associated methodologies. Health Inf Libr J. 2009;26(2):91-108.

24. Munn Z, Peters MDJ, Stern C, Tufanaru C, McArthur A, Aromataris E. Systematic review or scoping review? Guidance for authors when choosing between a systematic or scoping review approach. BMC Med Res Methodol. 2018;18(1):143.

25. Levaillant M, Marcilly R, Levaillant L, Vallet B, Lamer A. Assessing the hospital volume-outcome relationship in surgery: a scoping review protocol. BMJ Open. 2020;10(10):e038201

26. Arksey H, O'malley LSS. Scoping studies: towards a methodological framework; 2005

27. Levac D, Colquhoun H, O'Brien KK. Scoping studies: advancing the methodology. Implement Sci. 2010;5:69.

28. Tricco AC, Lillie E, Zarin W, O'Brien KK, Colquhoun H, Levac D, et al. PRISMA extension for scoping reviews (PRISMA-SCR): checklist and explanation. Ann Intern Med. 2018;169(7):467.

29. Chapter 11: Scoping reviews - JBI Reviewer's Manual - JBI GLOBAL WIKI. [cited 2019 Jul 31]. Available from: https://wiki.joannabriggs.org/display/ MANUAL/Chapter+11\%3A+Scoping+reviews

30. World Health Organization. The ICD-10 classification of mental and behavioural disorders: clinical descriptions and diagnostic guidelines. Geneva; 1992. [cited 2020 Dec 29]. Available from: (http://www.who.int/ classifications/icd/en/bluebook.pdf

31. Maloy C. Library Guides: Data Resources in the Health Sciences: Clinical Data. [cited 2020 Feb 18]. Available from: https://guides.lib.uw.edu/hsl/ data/findclin

32. Charlson ME, Pompei P, Ales KL, MacKenzie CR. A new method of classifying prognostic comorbidity in longitudinal studies: development and validation. J Chronic Dis. 1987;40(5):373-83.

33. Hurst JW, Morris DC, Alexander RW. The use of the New York heart Association's classification of cardiovascular disease as part of the patient's complete problem list. Clin Cardiol. 1999;22(6):385-90.

34. Elixhauser A, Steiner CA, Harris DR, Coffey RM. Comorbidity measures for use with administrative data. Med Care. 1998;36(1):8-27.

35. Saklad M. Grading of patients for surgical procedures. Anesthesiology. 1941;2(3):281-4.

36. Cavalcanti PEF, Sá MPBDO, dos Santos CA, Esmeraldo IM, Chaves ML, Lins RF d A, et al. Stratification of complexity in congenital heart surgery: comparative study of the risk adjustment for congenital heart surgery (RACHS-1) method, Aristotle basic score and Society of Thoracic Surgeons-European Association for Cardio-Thoracic Surgery (STS-EACTS) mortality score. Rev Bras Cir Cardiovasc. 2015;30(2):148-58.

37. Kellgrend J, Lawrence J. The epidemiology of chronic rheumatism. Atlas of standard radiographs, vol. 2. Oxford: Blackwell Scientific; 1963.

38. Prat J, FIGO Committee on Gynecologic Oncology. Staging classification for cancer of the ovary, fallopian tube, and peritoneum. Int J Gynaecol Obstet. 2014;124(1):1-5

39. Fuhrman SA, Lasky LC, Limas C. Prognostic significance of morphologic parameters in renal cell carcinoma. Am J Surg Pathol. 1982;6(7):655-63.
40. Baker SP, O'Neill B, Haddon W, Long WB. The injury severity score: a method for describing patients with multiple injuries and evaluating emergency care. J Trauma. 1974;14(3):187-96.

41. Rao PS, Schaubel DE, Guidinger MK, Andreoni KA, Wolfe RA, Merion RM, et al. A comprehensive risk quantification score for deceased donor kidneys: the kidney donor risk index. Transplantation. 2009;88(2):231-6.

42. Harsha SS, Archana BR. SNAPPE-II (score for neonatal acute physiology with perinatal extension-II) in predicting mortality and morbidity in NICU. J Clin Diagn Res. 2015:9(10):SC10-2.

43. Huntington SP. The clash of civilizations and the remaking of world order. New York: Touchstone; 1997.

44. Les établissements de santé - édition 2019 - Ministère des Solidarités et de la Santé. [cited 2020 Nov 7]. Available from: https://drees.solid arites-sante.gouv.fr/etudes-et-statistiques/publications/panoramas-dela-drees/article/les-etablissements-de-sante-edition-2019\#Texte-integral

45. Les cancers en France : l'essentiel - édition 2019 - ONCORIF. [cited 2020 Nov 7]. Available from: https://www.oncoriffr/les-cancers-en-france-lesse ntiel-de-2019/

46. Surgery statistics. [cited 2020 Dec 3]. Available from: https://stanfordhe althcare.org/medical-clinics/surgery-clinic/patient-resources/surgerystatistics.html

47. Gusmano M, Rodwin V, Weisz D, Cottenet J, Quantin C. Comparison of rehospitalization rates in France and the United States. J Health Serv Res Policy. 2015;20(1):18-25.

48. Arora S, Keeley J, Pucheril D, Menon M, Rogers CG. What is the hospital volume threshold to optimize inpatient complication rate after partial nephrectomy? Urol Oncol: Semin Orig Investig. 2018:36(7):339.e17-23.

49. Bernard A, Cottenet J, Mariet A-S, Quantin C, Pagès P-B. Is an activity volume threshold really realistic for lung cancer resection? J Thorac Dis. 2018;10(10):5685-94.

50. Beal EW, Mehta R, Hyer JM, Paredes A, Merath K, Dillhoff ME, et al. Association between travel distance, hospital volume, and outcomes following resection of cholangiocarcinoma. J Gastrointest Surg. 2019;23(5):944-52.

51. Mukhtar RA, Kattan OM, Harris HW. Variation in annual volume at a university hospital does not predict mortality for pancreatic resections. HPB Surg. 2008;2008:1-6.

52. Langabeer JR, Kim J, Helton J. Exploring the relationship between volume and outcomes in hospital cardiovascular care. Qual Manag Health Care. 2017;26(3):160-4

53. Avdic D, Lundborg P, Vikström J. Estimating returns to hospital volume: evidence from advanced cancer surgery. J Health Econ. 2019;63:81-99.

54. Liu JB, Bilimoria KY, Mallin K, Winchester DP. Patient characteristics associated with undergoing cancer operations at low-volume hospitals. Surgery. 2017;161(2):433-43.

55. Stitzenberg KB, Sigurdson ER, Egleston BL, Starkey RB, Meropol NJ. Centralization of cancer surgery: implications for patient access to optimal care. JCO. 2009;27(28):4671-8.

56. Ward MM, Jaana M, Wakefield DS, Ohsfeldt RL, Schneider JE, Miller T, et al. What would be the effect of referral to high-volume hospitals in a largely rural state? J Rural Health. 2004;20(4):344-54.

57. Dimick JB, Birkmeyer NJ, Finks JF, Share DA, English WJ, Carlin AM, et al. Composite measures for profiling hospitals on bariatric surgery performance. JAMA Surg. 2014;149(1):10.

58. Bramer WM, Rethlefsen ML, Kleijnen J, Franco OH. Optimal database combinations for literature searches in systematic reviews: a prospective exploratory study. Syst Rev. 2017;6(1):245.

\section{Publisher's Note}

Springer Nature remains neutral with regard to jurisdictional claims in published maps and institutional affiliations. 